\title{
Pasteurization of Milk
}

\author{
By Prof. G. S. Wilson
}

$\mathrm{T}$ EN, or even five, years ago there was a very real cleavage of opinion among scientific workers in Great Britain on the value of pasteurization. In general, the physiologists and biochemists were afraid of the possible damage caused by heat to the nutrient substances in the milk, and were inclined to minimize the part played by milk in the spread of infective disease. Bacteriologists and public health workers, on the other hand, were becoming increasingly impressed with the danger of drinking raw milk, and, in the absence of any convincing evidence to show that the nutritive value of the milk was seriously damaged by mild heat treatment, they strongly favoured the extended use of pasteurization as a means of diminishing the amount of preventable disease.

To-day this cleavage of opinion is much less apparent-indeed, it would scarcely be an exaggeration to say that it no longer exists. The change has come about, as all similar changes in scientific opinion must do, as the result of increased knowledge. During the past few years, several studies have been made on rats, mice and particularly calves, which have agreed in showing that only a minimal amount of damage is caused to the nutritive value of milk by low-temperature pasteurization. In addition, the occurrence of repeated outbreaks of milk-borne disease, some of them accompanied by a relatively high mortality, has gone a long way to convince unprejudiced observers that tuberculosis is not by any means the only infection spread by milk to human beings. It is now recognized that cleanliness is not enough. The milk must also be safe-safe from the risk of spreading disease-if it is to be recommended more freely to supplement the inadequate or unbalanced dietary of certain sections of our population. In view of this increasing unanimity, it will probably be better to give a brief résumé of the evidence on which our present conclusions are based rather than to set out the pros and cons of pasteurization $^{1}$, and thus create the illusion of a difference of opinion that now no longer exists. For reasons of space, discussion will be confined to the nutritional and hygienic aspects of cows' milk pasteurized by the official holding process $\left(145^{\circ}-150^{\circ} \mathrm{F}\right.$. for 30 minutes, followed by cooling to $55^{\circ} \mathrm{F}$. or below) and sold under licence. Not until these two aspects are fully understood is it profitable to consider the economic implications of compulsory pasteurization which play so large a part in practice in determining opposition to this measure.

The observations of numerous workers ${ }^{2,3}$ agree in showing that pasteurization has the following effects. About 5 per cent of the lactalbumin is coagulated; the coagulation time by rennin is lengthened, but the casein clot formed consists of finer particles and is of a more open texture; there is a diminution in the soluble calcium and phosphate, estimated variously by different workers, but probably of the order of 5 per cent; there may be some loss of iodine through volatilization; there is some destruction, at present unmeasured but apparently small, of vitamin $\mathbf{B}_{1}$; and there is some diminution in the vitamin $\mathrm{C}$ content, depending largely on the amount of previous exposure to light and on the presence of copper in the pasteurizing plant. Information on the effect of pasteurization on vitamin $D$ is still deficient, but considering that vitamin $D$ is even more resistant to heat and oxidation than vitamin A, it is improbable that any deleterious effect occurs. As against these positive changes may be set a number of negative findings. Thus it is found from feeding experiments on rats and calves that the biological value of the proteins is unaltered, that there is no detectable change in the availability of calcium or phosphorus, that there is no loss of vitamin A, and that the total energy value of the milk remains unaltered. Furthermore, recent carefully designed experiments on calves ${ }^{4,5}$ in which alternate animals were fed for 8-12 weeks on raw and pasteurized milk from the same source, have failed to show any significant difference in growth between the two groups of animals. In each experiment the average percentage weight increase was slightly higher in the group of animals receiving the pasteurized supply, but the differences were such as might easily have occurred by chance variation alone.

In assessing these results in terms of human nutrition it must be remembered that, though cows' milk may be a perfect food for calves, it is not a perfect food for infants. It differs both qualitatively and quantitatively from human milk, and it is deficient in iron, copper, vitamin $\mathrm{C}$, and often in vitamin $\mathrm{D}$. The differences between raw and pasteurized cows' milk are very much less than those between cows' milk and human milk. Deductions, therefore, from animal experiments to 
man must be made with great caution. Unfortunately, there is a dearth of direct observations to determine the comparative nutritive value of raw and pasteurized milk for infants and children. The only experiment of any size that is on record ${ }^{6}$ yielded results which failed to show any significant differences between the two classes of milk. Pending the publication of further observations of this type, we must conclude on the basis of all the available evidence that the nutritive changes produced by pasteurization appear to be comparatively slight, and are such as can readily be made good by suitable supplements to the dietary.

Turning now to the hygienic aspect of pasteurization, we have at our disposal well-documented evidence to show the danger of drinking ordinary raw milk and the protection afforded by adequate pasteurization against milk-borne disease. It is calculated' that in 1931 there were 2,600 deaths in Great Britain from tuberculosis of bovine origin and more than 6,000 fresh cases of the disease. Since then our knowledge of the part played by the bovine tubercle bacillus in causing pulmonary tuberculosis has considerably expanded, and there is no doubt that if this calculation were to be made afresh the figures would have to be increased rather than diminished. Undulant fever is a less important disease, but something like 400 cases yearly appear to arise in Great Britain, mainly from milk-borne infection ${ }^{1}$. Of epidemic diseases records ${ }^{1}$ are available of the occurrence in Great Britain during the years $1912-35$ of at least 103 milk-borne outbreaks of scarlet fever, septic sore throat, diphtheria, typhoid fever, paratyphoid fever, dysentery and gastro-enteritis affecting over 11,000 persons. These figures take no account of epidemic diarrhoea, in the causation of which milk almost certainly plays a considerable part. Our knowledge of sporadic milk-borne disease, apart from tuberculosis and undulant fever, is very deficient, since milk is rarely suspected as a vehicle of infection unless large numbers of cases occur in a short interval of time over a limited area, but as further information accumulates on this subject, it seems likely that the case against raw milk will have to be weighted still more heavily.

Pasteurization, adequately carried out in properly designed apparatus, and conveniently controlled in practice by the phosphatase test, destroys all pathogenic organisms in milk and renders it safe for human consumption. The laboratory and field evidence for these statements is too extensive to quote in detail. Reference may, however, be made to the now well-known observations of Price $^{8}$ in Toronto, which have all the force of an ad hoc experiment. Briefly, she found that among 300 tuberculous children bacteriologically examined,
15 per cent were infected with the bovine tubercle bacillus. All of these had come from parts of Ontario where pasteurization of milk was not carried out. In Toronto itself, where pasteurization has been compulsory since 1915, not a single case of bovine infection was found, in spite of the fact that 26 per cent of the incoming pooled supply of raw milk before pasteurization contained living tubercle bacilli. Nearer home we may add the recent experience at the Hannah Dairy Research Institute gained during one of the calffeeding experiments alluded to above ${ }^{5}$ of 36 calves fed for twelve weeks on raw milk, no fewer than 24 were found, when slaughtered, to be infected with the tubercle bacillus and to have macroscopic lesions of tuberculosis, whereas of 37 calves fed on milk from the same source after holder pasteurization, not a single one appeared to be infected. Apart from tuberculosis, the value of pasteurization in protecting against epidemic disease is well demonstrated by experience in the United States. Examination of the statistical returns shows that milk-borne disease has been practically eliminated from the large cities, where the great majority of the milk is pasteurized, and is now almost entirely confined to the rural districts and smaller towns, where most of the milk is consumed raw ${ }^{9}$. The effect of pasteurization in bringing to an end milk-borne epidemics that are already under way has been frequently and forcibly illustrated during the course of the past few years in Great Britain, notably in the extensive outbreak of typhoid fever at Bournemouth in $1936^{10}$.

In view of this cumulative evidence it is not surprising that public health workers are asking for the control of milk-borne disease by compulsory pasteurization. From the hygienic point of view, this step is merely the equivalent to the treatment of water by filtration and chlorination. Recent experience has shown what widespread public disapprobation follows neglect to exercise adequate control over our water supply, and there seems no logical reason why the public conscience should not be similarly exercised over the safety of our milk supply.

It has been suggested that the right way to control milk-borne infection is to eradicate disease from our milking herds. While not depreciating for one moment the importance of this aim, it must be pointed out that pathogenic organisms may gain access to milk not only from the cow, but also from the human personnel, from the water supply, and possibly from rodents. Even if eradication of cattle disease within a generation or two were possible, it would still leave unsolved the problem of protecting the milk supply from these other sources of contamination. 
Pasteurization, preferably accompanied by automatic bottling, alone affords an immediate, practicable, and effective method of ensuring the final safety of the milk as delivered to the consumer.

So far as the available evidence goes, there is no scientific argument against pasteurization. That the introduction of compulsory pasteurization might have widespread economic repercussions may be true, but it is not the purpose of this article to deal with a problem which comes into the domain of practical politics rather than of science. It is enough to show that, provided minor additions are made to the dietary of infants whose sole source of nourishment is pasteurized cows' milk-additions which are almost equally necessary on a dietary of raw cows' milk-there is no reason to fear that the growth and general development of our population would be in any way prejudiced by the extended use of pasteurized milk, while there is every reason to believe that the introduction of compulsory pasteurization, carried out under proper supervision, would lead to the practical abolition of milk-borne disease.

\footnotetext{
${ }^{1}$ Bendixen, H. C., Blink, G. J., Drummond, J. C., Leroy, A. M. and Wilson, G. S., Quart. Bull. Health Org. League of Nations, 6, 371 (1937).

${ }^{2}$ Stirling, J. D., and Blackwood, J. H., Hannah Dairy Res. Bull., No. 5 (1933).

'Report on "Milk and Nutrition", Nat. Inst. Res. Dairying, Reading and Rowett Res. Inst. Aberdeen. Poynder and Son, Reading (1937). 'Wilson, G. S., Minett, F. C., and Carling, H. F., J. Hyg., Camb. 37, 243 (1937).

${ }^{5}$ Wilkie, J., Edwards, S. J., Fowler, A. B., and Wright, N. C., J. Dairy Res., 8, 311 (1937). "Leighton, G., and McKinlay, P. L., "Milk Consumption and the
Growth of School Children", Dept. Health Scotland. H.M. Stationery Growth of School Children
Office, London (1930).

'Report Econ. Advis. Coun., Comm. Cattle Dis. H.M. Stationery Office, London (1934).

8 Price, R. M. Canad. Pub. Health J., 25, 13 (1934).

- Pub. Health Report, Wash., 46, 769 (1931).

${ }^{10}$ Shaw, W. V., Rep. Pub. Health Med. Subj., Lond., No. 81 (1937).
}

\section{Root-Pressure as a Factor in the Rise of Sap*}

\section{By Dr. Philip R. White, Rockefeller Institute for Medical Research, Princeton}

T HE history of our observations and theoriesone can scarcely say our 'knowledge'-concerning the movement of sap in plants has been a rather tortuous one. Beginning with the 'vital' explanations of Hales ${ }^{1}$ and $\mathrm{Knight}^{2}$, in which the living cells were presumed to be the agents largely responsible for sap movement, our concepts have run the entire gamut to the 'mechanical' explanations of Dixon and Joly $^{3}$ and Askenazy ${ }^{4}$ in which living cells were presumed to take no part in, or even to hinder, themovement of sap. TheDixon-Askenazy scheme is the one generally considered to-day to furnish the most satisfactory explanation of sap movement, while 'root-pressure', first observed by Hales in 1727, is looked upon as a transitory phenomenon, involving negligible forces (maximum value recorded, about $1.5 \mathrm{~atm}$.), the occurrence of which in uninjured plants was subjected to doubt by. Molisch $^{5}$, and hence probably unimportant.

For a number of years, I have been growing excised roots in vitro, isolated from all the influences which the rest of the plant normally exert upon the root ${ }^{6,7}$. It has thus been possible to establish with a considerable degree of precision the nature of these influences. It has been shown that the tops of plants provide the roots not only with carbohydrates but also with amino-acids ${ }^{8}$ and with materials ${ }^{9}$ either identical with or capable of being replaced by at least one vitamin, $B_{1}$. On

- Substance of a paper presented on December 28, 1937, before the Physiological Section of the Botanical Society of America at the the Physiological Section of the Botanical Society of America at the annual meeting of the American Association for the Advancement of appreciated Force in Sap Movement". the other hand, roots are not absolutely dependent on the tops for hormones, as was once supposed. Most of these studies have been carried out on roots of tomato which have been maintained in continuous culture for about five years (250 passages). Roots of a number of other plants have also been studied. Those of aster (Callistephus hortensis Cass.) have been grown for about 100 passages, those of Nicotiana langsdorffii Wernm. for about 75 passages, those of Brassica nigra Koch, Trifolium repens L., Petunia violacea Lindl., Fagopyrum esculentum Moench, Daucus carota L., and Helianthus annuus $\mathrm{L}$. for about 50 passages and those of a dozen more species for lesser periods.

The capacity to grow as autonomous units seems to be a general property of roots, at least among the dicotyledons. Now, one of the striking and universal features of these root cultures is that they retain their morphological characteristics, their growth polarity and their vascular strands. These strands are normally developed, although the cultures are grown floating upon, or immersed in, the liquid nutrient. Since in such cultures no obvious function could be ascribed to such strands, their presence seemed anomalous. If, however, a continuous flow of liquid be assumed to take place through these roots, that is to say, if they continue to function in culture in the manner postulated by Hales, as pumps, then the presence of vascular strands would be understandable. The root cultures seemed to offer a possible means of answering the old question : "Is root-pressure a real function 